\section{Higher risk of long QT syndrome and atrial flutter in adults with HIV admitted for acute myocardial infarction}

To the Editor;

Patients with HIV are at increased risk of cardiac arrhythmias, and sudden cardiac death is considered the third common cause of death (1). However, little is known about the differences in arrhythmias seen in patients with HIV following acute myocardial infarction (AMI). Hence, we used the 2017 National Inpatient Sample (NIS) to study the outcomes of HIV patients admitted in the United States.

The NIS is one of the largest inpatient databases produced by The Agency for Healthcare Research and Quality (AHRO), Healthcare Cost and Utilization Project (HCUP), and their multiple partners (2). The database is available online at hcup-us.ahrq.gov/ nisoverview.jsp. Patients of ages 18 and above were included in our study. Hospitalizations with a primary diagnosis of AMI and a diagnosis of HIV were identified using their respective International Classification of Diseases, Tenth Revision (ICD-10) codes provided by $\operatorname{HCUP}(3,4)$. Cases with long QT syndromes, atrial flutter, paroxysmal atrial fibrillation, supraventricular tachycardia, and ventricular fibrillation were also identified. The number of cases presenting with each arrhythmia was calculated and further estimated as the number of cases per 10,000 HIV patients with and without HIV. Their p-values, odds ratios (ORs), and 95\% confidence intervals (Cls) were also estimated (4).

Our study found 662,055 hospitalized cases of AMI among adults in the United States in 2017, and 2,860 had a diagnosis of HIV $(0.4 \%)$. The incidences of long OT syndrome, atrial flutter, paroxysmal atrial fibrillation, supraventricular tachycardia, and ventricular fibrillation in patients with HIV were 105 cases per 10,000 patients with HIV, 297 cases per 10,000 patients with HIV, 350 cases per 10,000 patients with HIV, 192 cases per 10,000 patients with HIV, and 280 cases per 10,000 patients with HIV, respectively, while they were 48 cases per 10,000 patients without HIV, 230 cases per 10,000 patients without HIV, 899 cases per 10,000 patients without HIV, 233 cases per 10,000 patients without HIV, and 304 cases per 10,000 patients without HIV. The results were statistically significant for long OT syndrome and atrial flutter as they were $2.204(95 \% \mathrm{Cl}=1.536-3.164, \mathrm{p}<0.001)$ and 1.300 (95\% Cl=1.047-1.614, $\mathrm{p}=0.017$ ) times more likely in patients with HIV. However, our findings among cases of supraventricular tachycardia $(p=0.15)$ and ventricular fibrillation $(p=0.45)$ were not statistically significant. A lower OR of $0.367(95 \% \mathrm{Cl}=0.300-0.448$, $p<0.001)$ was found for paroxysmal atrial fibrillation in patients with HIV. The mortality rate among patients hospitalized with AMI was higher with a diagnosis of HIV $(4.6 \%$ with HIV and $2.8 \%$ without HIV, $p<0.001$ ).
Our findings could potentially answer many questions related to HIV care. Acquired long OT syndrome has previously been reported among patients with HIV patients owing to their ART regimen that could include drugs such as nucleoside reversetranscriptase inhibitors, protease inhibitors, and non-nucleoside reverse-transcriptase inhibitors (5-7). Hunt et al. (8) found that protease inhibitors may not independently increase the risk of long QT syndrome, but the presence of other HIV and non-HIVrelated factors can potentiate the risk (8). Physicians should, therefore, carefully monitor and report any pre-AMI and post-AMI arrhythmias and reconsider the risks and benefits of the current doses and therapy chosen for the patients. Hsu et al. (9) found that the risk of atrial flutter in patients with HIV could be associated with their viral load. It was, however, not possible for us to further investigate this aspect owing to some of the limitations of the HCUP database (9).

The higher mortality risk in our study for patients with HIV following an AMI confirms multiple previous reports. The presence of various risk factors, such as higher viral load leading to an inadequate immune response, estimated glomerular filtration rate below $30 \mathrm{~mL} / \mathrm{min} / 1.73 \mathrm{~m}^{2}$, elderly groups ( 52 and above), and even lower body-mass index (below 18.5), have all been found to increase the risk of mortality among patients with HIV patients (10).

Finally, despite some of the HCUP database limitations, our results provide a conclusive proof of the increased risk of long OT syndrome and atrial flutter among patients with HIV following AMI, highlighting the need for more vigilance in this group of patients.

Acknowledgment: We are grateful to HCUP, AHRQ, and their partners for sharing access to the database with us. (https://www.hcup-us.ahrq. gov/partners.jsp)

Kamleshun Ramphul (D), Nomesh Kumar ${ }^{1}$ (D), Renuka Verma² ${ }^{(D)}$, Yogeshwaree Ramphul ${ }^{3}$ (D), Petras Lohana ${ }^{1}$ (D),

Stephanie G. Mejias ${ }^{4}\left(\mathbb{D}\right.$, Jyotsnav Joynauth ${ }^{5}$ (i)

Department of Pediatrics, Shanghai Xin Hua Hospital, School of Medicine, Shanghai Jiao Tong University; Shanghai-China ${ }^{1}$ Liaquat University of Medicine and Health Sciences; JamshrooPakistan

${ }^{2}$ Guru Gobind Singh Medical College; Punjab-India

${ }^{3}$ Sir Seewoosagur Ramgoolam National Hospital; PamplemoussesMauritius

${ }^{4}$ Department of Pediatrics, University Iberoamericana (UNIBE); Santo Domingo-Dominican Republic

5Institute of Pediatrics, Zhejiang University; Hangzhou-China

\section{References}

1. Brouillette J, Cyr S, Fiset C. Mechanisms of Arrhythmia and Sudden Cardiac Death in Patients With HIV Infection. Can J Cardiol 2019; 35: 310-9. [Crossref]

2. HCUP National Inpatient Sample (NIS). Healthcare Cost and Utilization Project (HCUP). 2017. Agency for Healthcare Research and Quality, Rockville, MD. Available from: URL; https://www.hcupus.ahrq.gov/nisoverview.jsp 
3. Ramphul K, Ramphul Y, Sombans S, Lohana P, Joynauth J. Acute myocardial infarction admissions among young adults in the United States: an update on the incidence and burden. Arch Med Sci Atheroscler Dis 2021; 6: e18-20. [Crossref]

4. Gupta A, Wang Y, Spertus JA, Geda M, Lorenze N, Nkonde-Price C, et al. Trends in acute myocardial infarction in young patients and differences by sex and race, 2001 to 2010. J Am Coll Cardiol 2014; 64: 337-45. [Crossref]

5. Chinello P, Lisena FP, Angeletti C, Boumis E, Papetti F, Petrosillo N. Role of antiretroviral treatment in prolonging QTc interval in HIVpositive patients. J Infect 2007; 54: 597-602. [Crossref]

6. Saquinavir: prolongation of PR and OT intervals. Prescrire Int 2011; 20: 17.

7. Castillo R, Pedalino RP, El-Sherif N, Turitto G. Efavirenz-associated QT prolongation and Torsade de Pointes arrhythmia. Ann Pharmacother 2002; 36: 1006-8. [Crossref]

8. Hunt K, Hughes CA, Hills-Nieminen C. Protease inhibitor-associated OT interval prolongation. Ann Pharmacother 2011; 45: 1544-50. [Crossref]
9. Hsu JC, Li Y, Marcus GM, Hsue PY, Scherzer R, Grunfeld C, et al. Atrial fibrillation and atrial flutter in human immunodeficiency virus-infected persons: incidence, risk factors, and association with markers of HIV disease severity. J Am Coll Cardiol 2013; 61: 2288-95. [Crossref]

10. Feinstein MJ, Nance RM, Delaney JAC, Heckbert SR, Budoff MJ, Drozd DR, et al. Mortality following myocardial infarction among HIV-infected persons: the Center for AIDS Research Network Of Integrated Clinical Systems (CNICS). BMC Med 2019; 17: 149. [Crossref]

Address for Correspondence: Kamleshun Ramphul, MD,

Department of Pediatrics, Shanghai Xin Hua Hospital, Shanghai Jiao Tong University, School of Medicine, 1665 Kongjiang Road, 200092, Shanghai-China Phone: +86 2165792951

E-mail: adramphul@hotmail.com (C) Copyright 2021 by Turkish Society of Cardiology Available online at www.anatoljcardiol.com DOI:10.5152/AnatolJCardiol.2021.222 